\title{
IoT Controlled All Terrain Rocker Bogie Robot
}

\author{
Prasath Kumar.S, Auvai Saraswathy.M, Malligeshwari.H, Nandhinni.Su
}

\begin{abstract}
In today's world, we concentrate mainly on newly emerging technologies for several monitoring, surveillance and recovery operations. This paper presents combination of two emerging technologies, which are Robotics and IoT. Most surveillance and monitoring robots does not have the ability to move on uneven surfaces and on slopes, but the rocker bogies have these features. While the present rocker bogies are remote controlled, it needs a human to be near it to control it. So our aim is to design a rocker bogie robot that can be controlled via IoT from a distance, which can be done using web page controlling. The control mechanism is provided with video transmission facility through high speed image transmission. The robot is fitted with a camera which captures the scene and transfer the images to the server on which the user can control and watch the live feed. We present the design of rocker bogie suspension and how to control it using commands in the further sections.
\end{abstract}

Keywords: Robotics, IoT, Rocker Bogie Suspension, Live feed, Web page controlling.

\section{INTRODUCTION}

Surveillance is essential in many fields for monitoring and providing accurate information about the status of a place which is prone to illegal entries of spies. Now-a-days as technology improves, robots are being used for monitoring and surveillance applications. These robots have a camera fitted to them which displays the scenes captured by live streaming to the user. But, there are several disadvantages which include the inability of these robots to move on uneven surfaces and slopes. This is overcome by rocker bogie suspension setup which is capable of moving in all types of uneven surfaces and terrains.

Rocker bogie suspension is nothing but a combination of a rocker and a bogie where bogie means the wheels of the robot and bogie means the connecting link between the bogies. This setup allows the robot to move on obstacles which are up to twice the diameter of the wheels.

Existing Rocker bogies are either remote controlled or based on artificial intelligence. The main drawback of remote controlled rocker bogies is it needs a human to control it within its nearby range which cannot make humanless monitoring possible. The disadvantage of artificial intelligence based rocker bogie is it cannot be controlled in

Revised Manuscript Received on December 22, 2018.

Prasath Kumar.S, Assistant professor, Department of Electronics and Communication Engineering, Sri Sairam Institute of Technology, Tamil Nadu, India

Auvai Saraswathy.M, UG scholor, Department of Electronics and Communication Engineering, Sri Sairam Institute of Technology,Tamil Nadu, India.

Malligeshwari.H, UG scholor, Department of Electronics and Communication Engineering, Sri Sairam Institute of Technology,Tamil Nadu, India.

Nandhinni.Su UG scholor, Department of Electronics and Communication Engineering, Sri Sairam Institute of Technology,Tamil Nadu, India. desired direction. It makes its automatic moves and cannot be controlled by the user.

To overcome all this problems, rocker bogie robot can be setup with IoT controlling section which would make the robot to traverse in the user desired direction as well as avoid any steeps present in the moving path. This makes the robot move even in slopes of 45 degrees and return without falling.

\section{OVERVIEW}

The proposed rocker bogie robot controlled using IoT takes commands from the webpage where the scenes captured by the robot are displayed. The webpage is divided into two sections. The section on the right side shows the scenes captured by the robot through live streaming. The session on the left has control buttons for the user to operate the robot from long distances.

The control section is written in HTML to place the buttons on the correct position.HTML is the main language used to build the webpage which use Php for traversing from the main page to the button status page. Clicking on the button changes the status of the button page which gives the control commands to the Raspberry pi3 which is present in the rocker bogie robot.

The Raspberry pi is the main component present in the rocker bogie setup which gets the command from the webpage and processes it and sends it to the motor driver IC. It does it through built in wifi modules for the access of the commands. The Raspberry pi used in this setup is of model B with quad core 64 bit ARM cortex A53 which is clocked at 1.2Ghz.We use Raspberry pi3 instead of Raspberry pi2 because it is $50 \%$ faster.

The motor driver IC gets the command from the Raspberry pi and controls the motors based on the command. The motor driver IC is L293D which is 16 pin IC with supply voltage 5 volts and $600 \mathrm{~mA}$ output current capability. It has two voltage pins one is used draw current for the working of L293D and other is for applying voltage for motors. It allows DC motor to drive on either directions simultaneously. We use L293D IC because it has internal ESD protection and high noise immunity inputs.

The dc motor driven by the driver runs at $100 \mathrm{rpm}$ which is basically a 12 volt DC motor. The rocker bogie has 6 wheels and connecting links acting as the rockers The whole setup is supplied with a 12volt-1A sealed rechargeable lead battery. The Raspberry pi takes commands written in Python which is recent and easiest coding language. This project requires XAMPP Php interpreter for interpreting the scripts written in the Php and Pearl language. It is a free and open source cross platform for the webserver. The tight VNC software is used to project the scenes captured by the robot as live

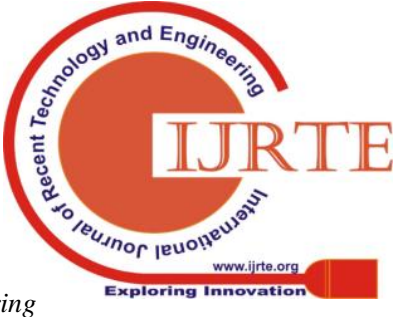
\& Sciences Publication 
feed.

\section{SYSTEM CONFIGURATION}

The block diagram given below shows the connection of

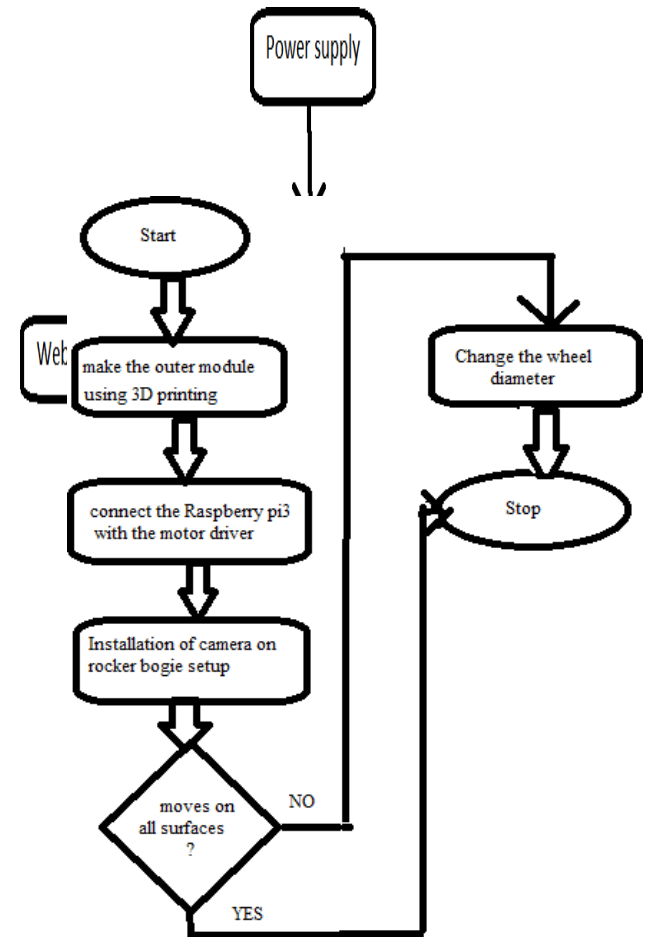

motor driver to motor and its wheels which are connected to the Raspberry pi3 installed with a camera. The whole setup is provided with the power supply.

Fig: Block diagram of rocker bogie setup Make the outer module using $3 \mathrm{D}$ printing

Initially, the diameter of the wheels is chosen based on the size of the obstacles it has to climb. The diameter is analysed and 6 wheels are made on about this diameter. The rocker is $3 \mathrm{D}$ printed and connected to this wheels. The bridge for connecting these rocker is designed and placed above them .The wheels are fitted with DC motors each which makes the wheels to move in desired direction.

Connect the Raspberry pi3 with the motor driver The Raspberry pi3 is tested and placed on the bridge of the rocker .It is connected to motor driver L294D along with battery. The motor driver is connected to the dc motor of the wheels. All the positive terminals are combined and given to the driver and negatives are grounded. The batteries negative terminal is grounded and positive terminal is connected to Raspberry pi

Installation of the camera over the rocker bridge

The webcam is mounted on the rocker bogie bridge and connected to the Raspberry pi through the ports. Initially the webcam is checked for resolution and focus and selected only if it is capable of live streaming in good quality. The webcam should be capable of capturing scenes with good resolution and even without daylight. The camera must be of high quality and high clarity. It must be checked prior to use. The camera must be fitted to correct port or else it will lead to faults.

Control the rocker bogie using webpage The webpage consisting of two sections is created using HTML. The button status is written using Php and XAMPP interpreter software is downloaded for interpreting Php. The monitoring section is governed by tight VNC software. It helps to provide live streaming video for the user to view.

Make the robot move on desired direction and slopes Now the rocker bogie robot is ready for verification in uneven terrain surfaces and slopes. The user should give the control commands using webpage and make the robot move in all directions. The robot is verified by moving on 45degree slopes. The robot can move on obstacles which are up to twice the diameter of wheels. If it needs any changes or improvements, the size of the wheels must be changed.

\section{DESIOGN FLOW}

The diagram given below represents the flowchart of the entire project.

Fig: flow chart of the design flow

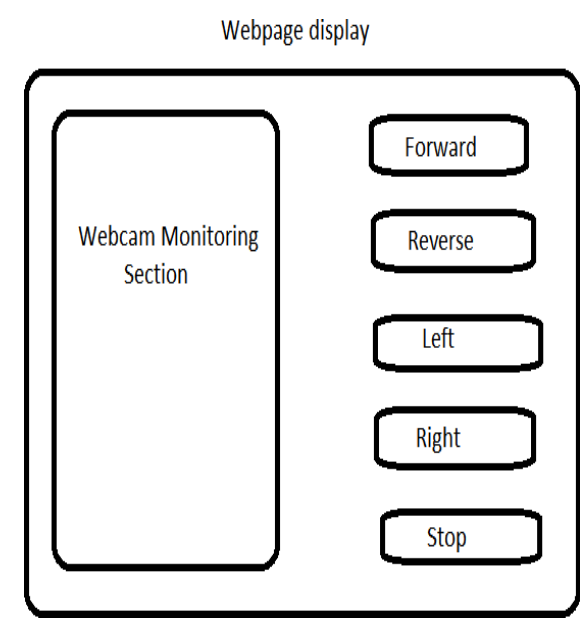

Fig: Webpage display

\section{COMPONENTS}

\section{Raspberry Pi 3 Model B}

It is a Quad core 64 bit ARM cortex A53 processor Clocked at $1.2 \mathrm{GHz}$ which has inbuilt Wi-fi. We use Raspberry pi3 because Pi 3 is 50\% faster than Pi 2.Raspberry pi3 is used instead of arduino because arduino does not have in-built wifi and Raspberry pi is good at software application rather than arduino which is good for hardware projects. Also pi is $40 \%$ faster than arduino at clock speed, and can run multiple programs at a time.

L293D motor driver

It is a 16 pin IC which has supply voltage of $5 \mathrm{~V}$ and 600 $\mathrm{mA}$ output current capability with pulsed current $1.2 \mathrm{~A}$. It allows DC motor to drive on either direction and can control a set of 2 DC motors simultaneously. It has internal ESD protection and high noise immunity inputs. It has 2 voltage pins, one is used to draw current for working of L293D and other is for applying voltage to motors. DC motor

It is a $12 \mathrm{~V} \mathrm{DC}$ motor that has $100 \mathrm{rpm}$ which can be run 
by the L293D motor driver. There are 6 wheels and hence there is a need for 6 DC motors.

Battery

It is a $12 \mathrm{~V} 1 \mathrm{~A}$ sealed rechargeable battery with cycle use of $4.4-15 \mathrm{~V}$ and stand-by use $13.5-13.8 \mathrm{~V}$ having initial current less than 0.39A.

Rocker Bogie setup

It needs 6 wheels of desired size, and 4 rockers along with a bridge for the components to be setup. It also needs a webcam to monitor and display the captured scenes. The webcam should be of high quality to display live streaming videos.

Fig: Sample webpage
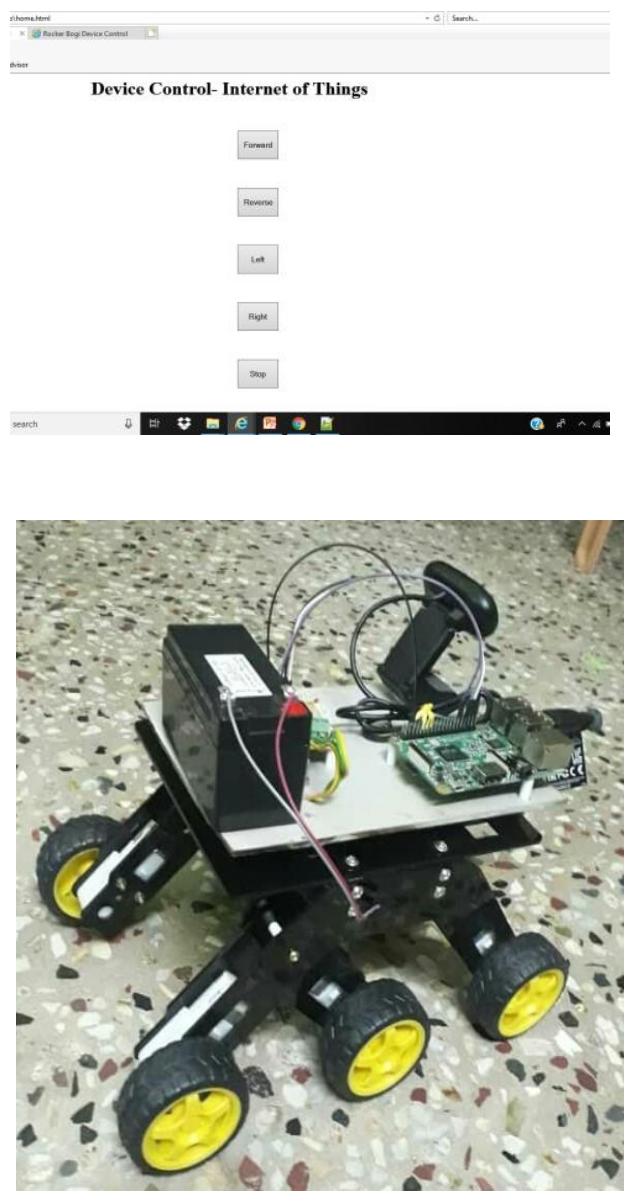

Fig: Rocker bogie setup

\section{RESULT}

The project is divided into 2 parts, one is the hardware part and the other is the software part. The hardware part consists of connecting the raspberry pi3 and the motor driver to the rocker bogie setup and the software part consists of controlling the device using IoT. Programs are written on HTML for creating the web page and the button status are created using Php. The raspberry pi is given commands on Python programming language.

\section{CONCLUSION}

The final output of the project is that it can move on all terrain surfaces and uneven areas and even in 45 degree slopes. It does not need human monitoring nearby to control it. Instead it can be controlled from far distances without any human support.

\section{REFERENCES}

1. Yunchuan Sun, Houbing Song, Antonio J. Jara, and Rongfang Bie, "Internet of Things and Big Data Analytics for Smart and Connected Communities”, Digital Object Identifier 10.1109/Access, March 2016.

2. Yuvraj Agarwal and Anind K. Dey, "Toward Building a Safe, Secure, and Easy-to-Use Internet of Things Infrastructure", IEEE Computer Society, April 2016.

3. Zhangbing Zhou, Beibei Yao, Riliang Xing, Lei Shu, and Shengrong Bu, "E-CARP: An Energy-Efficient Routing Protocol for UWSNs in the Internet of Underwater Things", IEEE Sensors Journal, Vol. 16, No. 11, June 2016

4. S.P. Raja, T. Dhiliphan Rajkumar and Vivek Pandiya Raj, Internet of Things: Challenges, Issues and Applications, Journal of Circuits, Systems and Computers, Vol. 27, No. 12, 2018

5. S.P. Raja, T. Sampradeepraj, Internet of Things: a Research oriented Introductory, International Journal of $\mathrm{Ad} \mathrm{Hoc}$ and Ubiquitous Computing, Vol. 29, No. 1/2, 2018.

6. Rajesh, M., and J. M. Gnanasekar. "Path Observation Based Physical Routing Protocol for Wireless Ad Hoc Networks." Wireless Personal Communications 97.1 (2017): 1267-1289.

7. Rajesh, M., and J. M. Gnanasekar. "Sector Routing Protocol (SRP) in Ad-hoc Networks." Control Network and Complex Systems 5.7 (2015): 1-4.

8. Rajesh, M. "A Review on Excellence Analysis of Relationship Spur Advance in Wireless Ad Hoc Networks." International Journal of Pure and Applied Mathematics 118.9 (2018): 407-412.

9. Rajesh, M., et al. "SENSITIVE DATA SECURITY IN CLOUD COMPUTING AID OF DIFFERENT ENCRYPTION TECHNIQUES." Journal of Advanced Research in Dynamical and Control Systems 18.

10. Rajesh, M. "A signature based information security system for vitality proficient information accumulation in wireless sensor systems." International Journal of Pure and Applied Mathematics 118.9 (2018): 367-387.

11. Rajesh, M., K. Balasubramaniaswamy, and S. Aravindh. "MEBCK from Web using NLP Techniques." Computer Engineering and Intelligent Systems 6.8: 24-26.

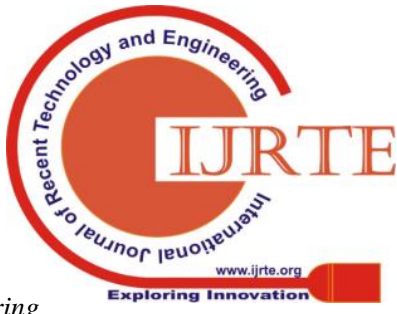

